Katharina Meyer ${ }^{1}$, Linda Rezny ${ }^{1}$, Christoph Breuer², Markus Lamprecht ${ }^{3}$, Hans Peter Stamm ${ }^{3}$

1 Swiss Health Observatory, Neuchâtel, Switzerland

2 Institute of Sports Sociology, Sports University, Cologne, Germany

${ }^{3}$ Lamprecht and Stamm, Social Science and Counceling, Zürich, Switzerland

\title{
Physical activity of adults aged 50 years and older in Switzerland
}

\author{
Submitted: 28 September 2004 \\ Accepted: 18 March 2005 \\ Published Online First: 21 July 2005
}

Summary

Objective: This study explores the prevalence of guideline-compliant physical activity (PA) behavior based on socio-demographic, health-related and environmental variables.

Methods: Cross-sectional data of the Swiss Health Survey 2002 were used in a Swiss population segment aged 50+. Four categories of PA were created with respect to Swiss and US guidelines: "moderate sports/exercise", "vigorous sports/exercise", "habitual PA", and "optimal PA" (i. e. combined variable used when both "vigorous sports/exercise" and "habitual PA" apply). Sedentary lifestyle was a fifth category.

Results: In the total sample considered for analysis ( $N=8405)$ the prevalence of guideline-compliant PA was: $9.1 \%$ moderate sports/exercise; $18.2 \%$ vigorous sports/exercise; $45 \%$ habitual PA; $8.7 \%$ optimal PA. Almost $50 \%$ of the age groups $65-79$ and $80+$ were involved in habitual PA. Higher socio-economic status was associated with involvement in moderate sports/exercise, vigorous sports/exercise, or optimal PA. In contrast, lower socioeconomic status was associated with habitual PA. While living in an urban or rural environment had no differentiating effect on sports/exercise, living in rural areas was associated with a sedentary lifestyle and little involvement in habitual PA and/or optimal PA. Self-reported good health and/or health orientation were associated with vigorous sports/exercise, habitual PA and optimal PA, regardless of whether the person had chronic health problems or not.

Conclusion: Habitual PA seems to have the greatest potential for increasing overall guideline-compliant activity levels. A behavioral/cognitive and political/environmental approach to improving PA is suggested.

Soz.- Präventivmed. 50 (2005) 218-229

Keywords: Guideline-compliant physical activity - Aging - Habitual physical activity - Strategies for promoting physical activity.

Keeping aging populations independent and free from disability is a major challenge in developed countries. Being a highly developed and economically stable country, Switzerland has one of the highest life expectancy rates in the world. However, there is generally a 6.5-year gap between "life expectancy", which is based on mortality statistics, and "healthy life expectancy".

Population models suggest that by focusing greater attention on reducing morbidity rather than mortality rates, it is possible to increase "healthy life expectancy" and reduce the period of disability in the latter years of life (Crimmins et al. 1994). Physical activity (PA) has been proven effective at reducing both morbidity and disability and at compensating for dysfunction among the chronically ill. PA is therefore considered a main factor in predicting whether a person will be free of disability in the latter years of his/her life (Vita et al. 1998).

Describing current PA behavior is a vital step towards promoting good exercise habits in an aging population. Empirical studies and theoretical models have shown that PA behavior patterns throughout one's life are influenced by various factors: physical and mental health status (AbuOmar et al. 2004a), perceived health (Abu-Omar et al. 2004b; Meyer et al. 2004), health orientation (Stewart et al. 1977) and mastery in a sense of self-efficacy (Booth et al. 2000) as well as social status, expressed e. g. by education and income, respectively (Lamprecht \& Stamm 2000). Moreover, physical environments play a large role in a person's choice to be physically active (Cunningham \& Michael 2004). These factors may also be associated with involvement in different modes and types of physical activity. 
Therefore, in order to adequately describe a population's PA behavior patterns, a distinction must be drawn between habitual physical activity (e.g. walking, cycling, household chores, etc.) and sports/exercise (e. g. aerobics workout, resistance training, swimming, etc.).

The purpose of this explorative study is to describe the prevalence of PA for more than 9000 Swiss citizens aged 50-80+. Self-reported PA behavior was used to create categories based on Swiss and US guidelines on exercise to improve fitness and health (American College of Sports Medicine 2000; Pate et al. 1995; Swiss Federal Office of Sports et al. 2000). Prevalence of guideline-compliant PA was determined for three age groups (50-64; 65-79, and +80 years). Moreover, the relationship between PA behavior and such factors as socio-demographic characteristics, economic conditions and health-related variables were also examined so as to further efforts to promote physical activity.

\section{Methods}

\section{Survey}

The present cross-sectional study is based on data from the Swiss Health Survey 2002 performed by the Swiss Federal Statistical Office (Swiss Federal Statistical Office 2003b). This population-based survey runs every five years. The Swiss Health Survey assesses self-reported socio-demographic features, physical/mental health status, disability and social consequences, use of the public health system, lifestyle and health-relevant behavior, living conditions, and health promotion aspects, among other things.

\section{Study population}

The sample was randomly selected from the Swiss permanent resident population aged 15 and older living in private residences connected to the telephone network. Data for the present study was taken from a survey conducted in 2002. The representative sample consisted of $\mathrm{N}=9171(\mathrm{~N}=5323$ males and $\mathrm{N}=3848$ females) aged $50+$, which corresponds to $46.5 \%$ of the total sample of 19706 respondents $(43.2 \%$ male and $49.3 \%$ females) surveyed in 2002 . Initially, we sorted through the $\mathrm{N}=9171$ sample to make sure that all of the members of the population sample fell into either one of the four physical activity categories or the fifth "sedentary lifestyle" category. This gave us the final population sample of $\mathrm{N}=8405$ for further analysis (see "Assessment of physical activity").

\section{Data collection}

Data were collected using computer-assisted telephone interviews (CATI, PROXY) and a written questionnaire. When- ever a respondent aged $=75$ could not or would not reply by CATI, a computer-assisted personal interview (CAPI) was arranged $(\mathrm{N}=205)$. Proxy interviews (PROXY) were used with close friends or relatives of respondents when the respondents themselves refused to answer questions, were unable to answer them because of a speech impediment, or were unavailable for a period of $4+$ weeks due to hospital stay, physical and/or mental incapacity $(\mathrm{N}=403) .83 \%$ of those interviewed by CATI or CAPI returned the written questionnaire. Data were collected consistently throughout the year (four rounds of data collection, each lasting three months) to prevent seasonal effects from having an impact on the assessed variables.

\section{Assessment of physical activity}

Sports/exercise: To establish the sports/exercise variable according to PA guidelines we considered three questions of the Swiss Health Survey. In terms of "intensity" subjects could choose between five levels of perceived exertion as indicated in the questionnaire. In terms of "duration" the subjects reported a number of minutes and/or hours per week they spent on sports/exercise. The variable sports/ exercise was based on information regarding the type of activity (i. e. calisthenics, fitness or sports), duration (hours per week) and perceived exertion. Calisthenics, fitness or sports activities at a perceived exertion of moderate and/or low for $\geq 180$ min per week were coded as "moderate sports/exercise". Moderate sports/exercise conforms to both CDC guidelines (Pate et al. 1995) and Swiss guidelines (Swiss Federal Office of Sports et al. 2000) (=150 min and >180 min per week, respectively, broken down into one 30 min session repeated five or more times per week).

Calisthenics, fitness or sports activities at a perceived exertion of quite strong or strong for $\geq 60$ min per week were coded as "vigorous sports/exercise". Vigorous sports/exercise conforms to ACSM guidelines (=60 min per week, broken down into $20 \mathrm{~min} /$ day sessions three or more times per week) (American College of Sports Medicine 2000). The categories "moderate sports/exercise" and "vigorous sport/ exercise" were mutually exclusive but each of them could overlap with the category "habitual PA (9.9\% of the habitually active subjects did also perform moderate sports/exercise, and $18.8 \%$ vigorous sports/exercise).

Habitual PA: This variable was established using information on usual daily walking and/or cycling assessed by two questions. If a subject did answer to walk and/or cycle habitually it was ask on how many minutes and/or hours per day. Moderate walking or cycling for 30 min per day was coded as "habitual PA". This activity category conforms to the PA 
recommendation made by the Swiss Federal Office of Sports (30 min per day $\geq 5$ times weekly) (Swiss Federal Office of Sports et al. 2000).

Optimal PA: This is a combined variable used when both vigorous sport/exercise and habitual $P A$ apply. It corresponds to $\geq 210 \mathrm{~min}$ of sports/exercise per week, which conforms to another Swiss recommendation (Swiss Federal Office of Sports et al. 2000).

No PA: This category refers to individuals who did no sports (be it moderate or vigorous) and additionally whose habitual PA was deemed insufficient ( $<30 \mathrm{~min} /$ day).

Groups that did sports/exercise below the intensities recommended in the guidelines $(\mathrm{N}=766)$ were not considered in the following analysis.

\section{Socio-demographic variables}

The following variables and categories were used: Males and females; age groups (50-64, 65-79 and 80+); education (primary, secondary, tertiary); size of household (one-person household, two and more person household); residence (urban: cities with > 100000 inhabitants or agglomerations with > 20000 inhabitants; rural); income in CHF (US\$): $<3000 \mathrm{CHF}$ (2 400 US\$), 3 000-4 500 (2 400-3 600), $>4$ 500-6000 (3 600-4 800), > 6000 (> 4 800)); language region (German-speaking, French-speaking, Italian-speaking part of Switzerland); socio-professional status (corporate manager; highly qualified non-manual worker; office clerk/ non-manual worker; small entrepreneur, self-employed; qualified manual worker; unqualified manual worker).

Self-reported health indicators (defined categories for analysis in parenthesis)

Body mass index: BMI $(<25 ; 25-30 ;>30)$. Health status variables were assessed with the questions as follows: perceived health status: "How is your health right now?"(very good/good; moderate; poor/very poor); perceived mental state: "How well-balanced is your state of mental health?" (not at all/not very; moderate; very/extremely); existence and/or absence of health problems/diseases in general and specifically: "Have you had any health problems/diseases in the past 12 months?" (yes/no); "Have you had any pain in the joints in the past four weeks?" (yes/no); "Have you undergone medical treatment for diabetes, hypertension and/or heart attack in the past 12 months?" (yes/no for each illness). Health orientation was assessed on the basis of two mutually exclusive items: unconcerned ("I live my life without worrying so much about my health") versus health-oriented ("thoughts about maintaining my health influence my lifestyle and health considerations have a major impact on the way I live"). Mastery in a sense of self-efficacy (variables used: unsolvable problems; carried by destiny; little control over circumstances; given up in the face of problems); parameter value: low; medium; high. Social support: membership in clubs (yes, no). The variables were chosen on an empirical and theoretical basis (see introduction).

\section{Statistical analysis}

Analysis was conducted using the SPSS program (Version 11). As a first step, socio-demographical variables and health-related variables were distributed for the defined PA categories. Prevalence of both "no PA" and "PA" categories was calculated at five-year age group intervals. The prevalence of physical activity for 50-64, 65-79 and 80+ age groups was also determined.

Using logistic regression analysis, the adjusted odds ratios and $95 \%$ confidence intervals (OR and 95\% CI) were calculated separately for the "no PA" category and the four "PA" categories (moderate sports/exercise, vigorous sports/ exercise, habitual PA, optimal PA), which were used as dependent variables. For socio-demographic variables, adjustment included all of the aforementioned variables plus socio-professional status. Health-related variables were adjusted for variables chosen on the basis of its empirical association to PA (see Tab. 3 and 4).

The data were weighted to get a sample matching the Swiss population in terms of important socio-demographic variables. Different weightings were used for data obtained from the telephone or written surveys. $P$ value of 0.05 was considered significant.

\section{Results}

\section{Physical inactivity according to five-year-age groups} in an aging Swiss population

More than one-third of the population assessed reported that they did neither sports/exercise nor sufficient habitual PA. With increasing age, the prevalence of physical inactivity takes on a U-form. Considered for five-year-age groups, sedentary lifestyle was most prevalent in individuals aged $\geq 80(39.3 \%)$ and lowest in the 65-69 age group (27.4\%) (Fig. 1). In all of the age groups, women had a lower prevalence of physical inactivity (range $23.9 \%-39.3 \%$ ) than men (range $31.1 \%-46.3 \%$ ) (Fig. 1; Tab. 1).

\section{Guideline-compliant physical activity}

Prevalence of guideline-compliant PA for the four categories assessed is presented in Fig. 2. Prevalence of moderate 
Physical inactivity (\%)

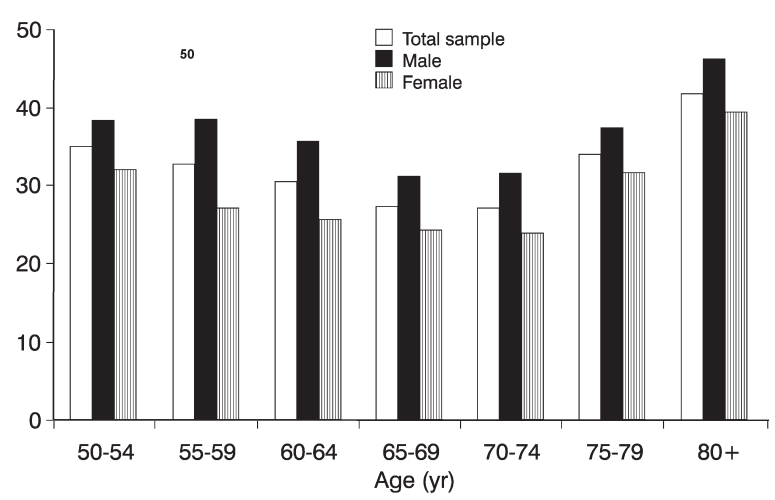

Figure 1 Physical inactivity by 5 yr group in $\mathrm{N}=9171$, permanent resident population in Switzerland

and vigorous sports/exercise and optimal PA decreased in direct proportion to increasing age. For habitual PA, the youngest age group (50-64 years) had the lowest prevalence, the oldest age group (age $\geq 80$ ) was in the middle and the 65-79 age group had the highest prevalence. With exception
Physical activity (\%)

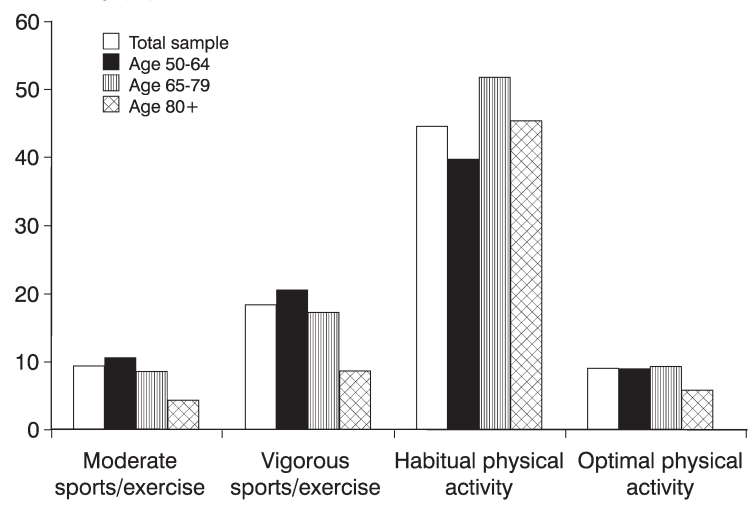

Figure 2 Prevalence of guideline-compliant physical activity by age group for each of the four categories

of moderate sports/exercise, more females did every single category of guideline-compliant PA than males. The prevalence of sports/exercise and habitual and optimal PA, respectively, according to socio-demographic, economic and health-related factors assessed are shown in Tab. 1 and 2.

Table 1 Percent meeting physical activity guidelines to improve physical fitness according to selected socio-demographic variables (Swiss Health Survey 2002)

\begin{tabular}{|c|c|c|c|c|c|c|}
\hline & $\begin{array}{l}\text { Overall } \\
\text { distribution } \\
\text { of variable } \\
\%(n)\end{array}$ & $\begin{array}{l}\text { No physical } \\
\text { activity }{ }^{\text {a) }} \\
\% \text { (n) }\end{array}$ & $\begin{array}{l}\begin{array}{l}\text { Moderate } \\
\text { sports/exercise }^{\text {a) }}\end{array} \\
\%(n)\end{array}$ & $\begin{array}{l}\begin{array}{l}\text { Vigorous } \\
\text { sports/exercise }\end{array} \\
\%(n)\end{array}$ & $\begin{array}{l}\text { Habitual physical } \\
\text { activity }^{\text {a) }} \\
\%(n)\end{array}$ & $\begin{array}{l}\text { "Optimal" physical } \\
\text { activitya) } \\
\% \text { (n) }\end{array}$ \\
\hline \multicolumn{7}{|l|}{ Age } \\
\hline $50-64$ & $53.6(4348)$ & $33(1518) * * *$ & $10.4(495) * * *$ & $20.5(1003)^{* * *}$ & $39.7(1983)^{* * *}$ & $8.9(430) * * *$ \\
\hline $65-79$ & $37.2(3257)$ & 29.1 (983) & $8.3(303)$ & $17.2(576)$ & $51.7(1900)$ & $9.2(324)$ \\
\hline $80+$ & $9.2(800)$ & 41.9 (335) & $4.3(39)$ & $8.4(87)$ & $45.4(414)$ & $5.7(55)$ \\
\hline Total & $100.0(8405)$ & $32.3(2836)$ & $9.1(837)$ & $18.2(1666)$ & $44.6(4297)$ & $8.7(809)$ \\
\hline \multicolumn{7}{|l|}{ Gender } \\
\hline Male & $45.1(3542)$ & $36.7(1372) * * *$ & $9.8(396)$ ** & $16.8(650)$ ** & $38.3(1554)$ *** & $5.8(259) * * *$ \\
\hline Female & $54.9(4863)$ & $28.8(1464)$ & $8.5(441)$ & $19.4(1016)$ & $49.9(2743)$ & $11.1(560)$ \\
\hline \multicolumn{7}{|l|}{ Education } \\
\hline Primary & $26.0(2233)$ & $42.5(953) * * *$ & $5.1(120)$ & $9.8(262) * * *$ & $43.6(2129)^{* * *}$ & $5.3(139) * * *$ \\
\hline Secondary & 60.1 (5014) & 29.5 (1541) & $10.0(562)$ & $20.3(1092)$ & $46.0(2648)$ & $10.0(542)$ \\
\hline Tertiary & $13.9(1144)$ & $25.8(333)$ & $12.5(155)$ & $24.7(311)$ & $41.2(535)$ & $9.7(128)$ \\
\hline \multicolumn{7}{|l|}{ Income (CHF) } \\
\hline$<3000$ & $31.5(2374)$ & $39.2(926) * * *$ & $6.6(168) * * *$ & $14.1(340) * * *$ & $44.4(1206)^{\star * *}$ & $6.7(169) * * *$ \\
\hline $3000-4500$ & $37.2(2819)$ & $29.1(880)$ & $9.3(281)$ & $18.5(586)$ & 48.4 (1539) & $9.8(304)$ \\
\hline $4500-6000$ & $20.5(1770)$ & $26.4(507)$ & $11.3(219)$ & $23.6(436)$ & $45.2(937)$ & $11.1(221)$ \\
\hline$>6000$ & $10.8(874)$ & $25.5(257)$ & $14.8(143)$ & $25.2(244)$ & $37.0(363)$ & $10.2(88)$ \\
\hline \multicolumn{7}{|l|}{ Size of household } \\
\hline 1 person & $22.5(3075)$ & $25.9(894) * * *$ & $8.5(284) * * *$ & $16.5(534)$ & $55.8(1814)^{* * *}$ & $9.3(299)$ \\
\hline$\geq 2$ persons & $77.5(5330)$ & $34.2(1942)$ & $9.3(553)$ & $18.7(1132)$ & $41.5(2483)$ & $8.6(510)$ \\
\hline \multicolumn{7}{|l|}{ Residence } \\
\hline Urban & $75.4(6268)$ & $29.7(1948) * * *$ & $9.4(1292)$ * & $19.1(645)$ ** & $47.4(3367)^{* * *}$ & $9.6(648) * * *$ \\
\hline Rural & $24.6(2137)$ & $40.6(888)$ & $8.2(192)$ & $15.5(374)$ & $36.1(930)$ & $6.1(161)$ \\
\hline \multicolumn{7}{|l|}{ Language region } \\
\hline German speaking & $72.8(5676)$ & $29.3(1682) * * *$ & $9.8(616) * * *$ & $20.3(1293) * * *$ & $46.7(3064) * * *$ & $10.1(653) * * *$ \\
\hline French speaking & 22.1 (2002) & $41.9(890)$ & $6.9(157)$ & $12.6(276)$ & $37.8(865)$ & $5.2(121)$ \\
\hline Italian speaking & $5.0(727)$ & $34.3(264)$ & $9.6(64)$ & $12.1(97)$ & $44.4(368)$ & $4.2(35)$ \\
\hline
\end{tabular}

$\mathrm{p}<* 0.01 ; * * 0.001 ; * * * 0.0001 ;$ a) as defined in the text 
Table 2 Percent meeting physical activity guideline to improve physical fitness according to selected health variables (Swiss Health Survey 2002)

\begin{tabular}{|c|c|c|c|c|c|c|}
\hline & $\begin{array}{l}\text { Overall } \\
\text { distribution } \\
\text { of variable } \\
\%(n)\end{array}$ & $\begin{array}{l}\text { No physical } \\
\text { activity }^{\text {a) }} \\
\%(n)\end{array}$ & $\begin{array}{l}\begin{array}{l}\text { Moderate } \\
\text { sports/exercise }^{\text {a) }}\end{array} \\
\%(n)\end{array}$ & $\begin{array}{l}\begin{array}{l}\text { Vigorous } \\
\text { sports/exercise }^{\text {a) }}\end{array} \\
\%(n)\end{array}$ & $\begin{array}{l}\text { Habitual physical } \\
\text { activity }^{\text {a) }} \\
\%(n)\end{array}$ & $\begin{array}{l}\text { "Optimal" physical } \\
\text { activity }{ }^{\text {a) }} \\
\%(n)\end{array}$ \\
\hline \multicolumn{7}{|l|}{ Self-reported health } \\
\hline \multicolumn{7}{|l|}{ Perceived health } \\
\hline very good/good & 77.8 (6529) & $29.2(2012) * * * *$ & $10.0(711) * * * *$ & $20.2(1448) * * * *$ & $45.9(3420) * * * *$ & $9.7(707) * * * *$ \\
\hline moderate & $16.2(1401)$ & $39.2(557)$ & $6.9(101)$ & 11.9 (177) & 43.5 (707) & $6.0(89)$ \\
\hline poor/very poor & $6.0(472)$ & $56.2(267)$ & $3.8[25]$ & $8.2(40)$ & 30.5 (168) & $2.6[13]$ \\
\hline \multicolumn{7}{|l|}{$\begin{array}{l}\text { Well-balanced } \\
\text { mental state }\end{array}$} \\
\hline not at all/little & $14.6(1091)$ & $33.0(397) * * *$ & $7.4(89)$ * & $16.3(189) * * *$ & $45.1(561)$ & $9.0(101)$ \\
\hline moderate & $20.3(1622)$ & $29.1(497)$ & $9.4(163)$ & $18.5(322)$ & $48.8(888)$ & 9.1 (157) \\
\hline much/very much & $65.0(4971)$ & $28.4(1492)$ & $9.8(521)$ & $19.8(1064)$ & $48.5(2699)$ & $9.7(531)$ \\
\hline \multicolumn{7}{|l|}{$\begin{array}{l}\text { Body mass index } \\
\text { (BMI) }\end{array}$} \\
\hline$<25$ & $50.6(4268)$ & $28.2(1252) * * * *$ & $10.0(467)$ ** & $21.1(953) * * * *$ & $47.7(2312) \S$ & $10.5(482) * * * *$ \\
\hline $25-30$ & 38.5 (3072) & 33.5 (1079) & $9.0(229)$ & $16.9(577)$ & $43.5(1537)$ & $7.8(270)$ \\
\hline$>30$ & $10.9(920)$ & $43.4(428)$ & $6.7(66)$ & $10.7(117)$ & $37.6(391)$ & $4.2(47)$ \\
\hline \multicolumn{7}{|l|}{ Health status } \\
\hline \multicolumn{7}{|l|}{$\begin{array}{l}\text { Presence of health } \\
\text { problems/disease } \\
\text { in past } 12 \text { months }\end{array}$} \\
\hline yes & $23.7(2045)$ & $41.0(859) * * * *$ & $6.7(159) * * * *$ & $13.3(293) * * * *$ & $39.8(948) * * * *$ & $6.1(136) * * * *$ \\
\hline no & $76.3(6358)$ & $29.7(1975)$ & $9.9(678)$ & $19.7(1373)$ & 46.1 (3349) & $9.5(673)$ \\
\hline \multicolumn{7}{|l|}{$\begin{array}{l}\text { Pain in joints } \\
\text { (last } 4 \text { wks) }\end{array}$} \\
\hline yes & $13.0(1121)$ & $42.0(473) * * * *$ & $6.7(86)$ ** & $14.4(158)^{* * * *}$ & $40.5(524)$ ** & $6.6(76) * * * *$ \\
\hline no & 87.0 (7279) & 30.9 (2359) & $9.5(751)$ & $18.8(1508)$ & $45.3(3772)$ & $9.0(733)$ \\
\hline \multicolumn{7}{|l|}{ Diabetes $^{\text {b) }}$} \\
\hline yes & $7.1(475)$ & $33.6(164) * *$ & $8.5(49)$ & $11.1(63)$ **** & $52.2(264)$ & $5.7(33)$ * * \\
\hline no & $92.9(6295)$ & $28.2(1878)$ & $9.7(665)$ & $19.7(1352)$ & $48.5(690)$ & $9.9(690)$ \\
\hline \multicolumn{7}{|l|}{ Hypertension ${ }^{\mathrm{b})}$} \\
\hline yes & $28.3(1947)$ & $29.1(619)$ * & $8.7(185)$ * & $16.0(325) * * * *$ & $51.0(1111)$ * & $8.5(180)$ ** \\
\hline no & 71.7 (4819) & $28.4(1419)$ & $10.0(529)$ & $20.3(1090)$ & 47.9 (2593) & $10.1(543)$ \\
\hline \multicolumn{7}{|l|}{ Heart attack ${ }^{\mathrm{b})}$} \\
\hline yes & $4.8(318)$ & $31.6(108)$ & $9.0(36)$ & $13.1(49)$ * & $50.2(173)$ & $7.3(31)$ \\
\hline no & $95.2(6450)$ & $28.5(1933)$ & $9.7(678)$ & $19.4(1365)$ & $48.7(3530)$ & $9.7(691)$ \\
\hline \multicolumn{7}{|l|}{$\begin{array}{l}\text { Health orientation } \\
\text { Importance of health } \\
\text { for life }\end{array}$} \\
\hline unconcerned & $7.8(523)$ & $45.8(253) * * * *$ & $3.9(22) * * * *$ & $9.3(60) * * * *$ & $38.6(220) * * * *$ & $2.7(20) * * * *$ \\
\hline health-orientated & $92.2(6109)$ & $27.0(1713)$ & $10.2(683)$ & $20.1(1349)$ & $49.6(3410)$ & $10.3(699)$ \\
\hline \multicolumn{7}{|l|}{ Mastery } \\
\hline low & $19.8(1138)$ & $33.9(408) * * * *$ & $8.9(115)$ & $17.5(202) * * * *$ & $43.4(567)$ * & $7.8(99)$ * \\
\hline medium & 35.4 (2039) & $27.6(601)$ & $11.3(248)$ & 20.4 (439) & 48.8 (1119) & $10.8(230)$ \\
\hline high & $44.7(2527)$ & $26.6(700)$ & $10.0(271)$ & $20.3(599)$ & $48.8(1384)$ & $9.9(293)$ \\
\hline \multicolumn{7}{|l|}{ Social support } \\
\hline \multicolumn{7}{|l|}{ Membership in clubs } \\
\hline yes & 41.1 (3397) & $23.9(873) * * * *$ & $11.8(456) * * * *$ & $25.5(966) * * * *$ & $45.9(1788)$ & $12.1(459) * * * *$ \\
\hline no & $58.9(5005)$ & $38.5(1960)$ & $7.2(381)$ & $12.9700)$ & 43.7 (2509) & $6.3(350)$ \\
\hline
\end{tabular}

$\mathrm{p}<* 0.05 ; * * 0.01 ; * * * 0.001 ; * * * * 0.0001$

a) As defined in the text

b) Underwent medial treatment in the past 12 months

Numbers $=30$ are presented in [ ] and indicate restricted validity 
Table 3 Adjusted odds ratios a) for meeting physical activity guidelines by selected socio-demographic variables (Swiss Health Survey 2002)

\begin{tabular}{|c|c|c|c|c|c|}
\hline & $\begin{array}{l}\text { No physical } \\
\text { activityb) }\end{array}$ & $\begin{array}{l}\text { Moderate } \\
\text { sports/exercise }^{\text {b) }}\end{array}$ & $\begin{array}{l}\text { Vigorous } \\
\text { sports/exercise }^{\text {b) }}\end{array}$ & $\begin{array}{l}\text { Habitual physical } \\
\text { activity }^{\text {b) }}\end{array}$ & $\begin{array}{l}\text { "Optimal" physical } \\
\text { activityb) }\end{array}$ \\
\hline & $\begin{array}{l}\text { Adjusted OR } \\
(95 \% \mathrm{Cl})\end{array}$ & $\begin{array}{l}\text { Adjusted OR } \\
(95 \% \mathrm{Cl})\end{array}$ & $\begin{array}{l}\text { Adjusted OR } \\
(95 \% \mathrm{Cl})\end{array}$ & $\begin{array}{l}\text { Adjusted OR } \\
(95 \% \mathrm{Cl})\end{array}$ & $\begin{array}{l}\text { Adjusted OR } \\
(95 \% \mathrm{Cl})\end{array}$ \\
\hline \multicolumn{6}{|l|}{ Age } \\
\hline $50-64$ & 1 & 1 & 1 & 1 & 1 \\
\hline $65-79$ & $0.78(0.70-0.87)$ & $0.95(0.81-1.12)$ & $0.85(0.75-0.96)$ & $1.61(1.45-1.77)$ & $1.19(1.00-1.40)$ \\
\hline $80+$ & $1.25(1.03-1.50)$ & $0.56(0.39-0.81)$ & $0.53(0.40-0.69)$ & $1.32(1.10-1.57)$ & $0.81(0.57-1.13)$ \\
\hline \multicolumn{6}{|l|}{ Gender } \\
\hline male & 1 & 1 & 1 & 1 & 1 \\
\hline female & $0.61(0.55-0.68)$ & $0.96(0.81-1.13)$ & $1.41(1.24-1.60)$ & $1.42(1.28-1.57)$ & $1.90(1.59-2.28)$ \\
\hline \multicolumn{6}{|l|}{ Education } \\
\hline primary & 1 & 1 & 1 & 1 & 1 \\
\hline secondary & $0.67(0.59-0.76)$ & $1.60(1.26-2.04)$ & $1.50(1.26-1.79)$ & $1.11(0.98-1.26)$ & $1.43(1.13-1-81)$ \\
\hline tertiary & $0.54(0.44-0.66)$ & $1.74(1.27-2.37)$ & $1.99(1.58-2.52)$ & $1.14(0.95-1.36)$ & $1.93(1.40-2.66)$ \\
\hline \multicolumn{6}{|l|}{ Income (CHF) } \\
\hline$<3000$ & 1 & 1 & 1 & 1 & 1 \\
\hline $3000-4500$ & $0.76(0.67-0.86)$ & $1.16(0.94-1.43)$ & $1.14(0.97-1.33)$ & $1.16(1.03-1.30)$ & $1.26(1.02-1.56)$ \\
\hline $4500-6000$ & $0.75(0.65-0.87)$ & $1.35(1.08-1.71)$ & $1.36(1.14-1.62)$ & $1.00(0.87-1.14)$ & $1.48(1.17-1.87)$ \\
\hline$>6000$ & $0.74(0.61-0.90)$ & $1.72(1.31-2.25)$ & $1.43(1.15-1.77)$ & $0.70(0.58-0.83)$ & $1.19(0.88-1.62)$ \\
\hline \multicolumn{6}{|l|}{ Size of household } \\
\hline 1 person & 1 & 1 & 1 & 1 & 1 \\
\hline$\geq 2$ persons & $1.24(1.11-1.39)$ & $1.06(0.90-1.25)$ & $1.29(1.13-1.46)$ & $0.71(0.64-0.78)$ & $1.16(0.97-1.37)$ \\
\hline \multicolumn{6}{|l|}{ Residence } \\
\hline urban & 1 & 1 & 1 & 1 & 1 \\
\hline rural & $1.26(1.12-1.41)$ & $0.99(0.83-1.18)$ & $0.89(0.78-1.02)$ & $0.75(0.68-0.84)$ & $0.78(0.64-0.95)$ \\
\hline \multicolumn{6}{|l|}{ Language region } \\
\hline German speaking & 1 & 1 & 1 & 1 & 1 \\
\hline French speaking & $1.76(1.57-1.98)$ & $0.72(0.59-0.87)$ & $0.56(0.49-0.65)$ & $0.69(0.62-0.77)$ & $0.53(0.43-0.65)$ \\
\hline Italian speaking & $1.26(1.06-1.51)$ & $0.91(0.69-1.21)$ & $0.56(0.44-0.71)$ & $0.85(0.72-1.01)$ & $0.37(0.25-0.56)$ \\
\hline
\end{tabular}

a) Adjusted for all other variables in this table, and for socio-profession category; ${ }^{\text {b) }}$ As defined in the text

Relationship between guideline-compliant physical activity and socio-demographic variables (Tab. 3)

With increasing age, the elderly population was less likely to do guideline-compliant moderate and vigorous sports/ exercise but more likely to do habitual PA. Compared to men, women were more likely to be active in three of the four PA categories as assessed. Compared to urban residents, inhabitants of rural areas were more likely to be physically inactive and less likely to do habitual PA and/or optimal PA. In contrast, living in an urban or rural environment had no differentiating effect on moderate and/or vigorous sports/exercise. Living in different language areas of Switzerland was associated with a different involvement in both sedentary lifestyle and categories of PA as assessed. The higher the education and income of the aging population, the more likely it was to do moderate sports/exercise, vigorous sports/exercise, or optimal PA. A reverse association was seen between income and habitual PA. Living in single households and households with two or more persons had a differentiating effect on habitual PA and vigorous sports/ exercise.

\section{Relationship between physical activity and health-related variables (Tab. 4)}

The elderly who reported that they were in poor health, overweight or obese were more likely to be physically inactive than the elderly who reported contrasting parameter values for the above variables. Those who reported good health, normal BMI and no chronic health problems/diseases were more likely to be associated with three of the four categories of PA (vigorous sports/exercise, habitual PA, optimal PA), after adjustment for possible confounders. As for moderate sports/exercise, it did no matter whether the self-rated health was good, moderate and/or poor. Existence and/or absence of pain in the joints had a differentiating effect on physical inactivity, vigorous sports/exercise and habitual PA. Previous history of heart attack had no impact on the likelihood of a person being sedentary or physically active. Those who reported having no diabetes and or high blood pressure tended to be those who did vigorous sports/exercise and optimal PA. Those who were less active were generally those who reported having these cardiovascular risk factors. After adjustment for socio-demographic 
Table 4 Adjusted odds ratios ${ }^{\text {a)-b) }}$ for meeting physical activity guideline by selected health variables (Swiss Health Survey 2002)

\begin{tabular}{|c|c|c|c|c|c|}
\hline & $\begin{array}{l}\text { No physical } \\
\text { activityb) }^{\text {b) }}\end{array}$ & $\begin{array}{l}\text { Moderate } \\
\text { sports/exercise }^{\mathrm{d}} \text { ) }\end{array}$ & $\begin{array}{l}\text { Vigorous } \\
\text { sports/exercise }^{\text {d) }}\end{array}$ & $\begin{array}{l}\text { Habitual physical } \\
\text { activity }^{\text {d) }}\end{array}$ & $\begin{array}{l}\text { "Optimal" physical } \\
\text { activityd) }\end{array}$ \\
\hline & $\begin{array}{l}\text { Adjusted OR } \\
(95 \% \mathrm{Cl})\end{array}$ & $\begin{array}{l}\text { Adjusted OR } \\
(95 \% \mathrm{Cl})\end{array}$ & $\begin{array}{l}\text { Adjusted OR } \\
(95 \% \mathrm{Cl})\end{array}$ & $\begin{array}{l}\text { Adjusted OR } \\
(95 \% \mathrm{Cl})\end{array}$ & $\begin{array}{l}\text { Adjusted OR } \\
(95 \% \mathrm{Cl})\end{array}$ \\
\hline \multicolumn{6}{|l|}{ Self-reported health } \\
\hline \multicolumn{6}{|l|}{ Perceived healtha) } \\
\hline very good/good & 1 & 1 & 1 & 1 & 1 \\
\hline moderate & $1.17(0.99-1.38)$ & $0.88(0.68-1.14)$ & $0.68(0.55-0.83)$ & $0.98(0.84-1.13)$ & $0.72(0.55-0.95)$ \\
\hline poor/very poor & $1.85(1.43-2.40)$ & $0.74(0.45-1.21)$ & $0.52(0.34-0.80)$ & $0.69(0.53-0.89)$ & $0.44(0.24-0.83)$ \\
\hline \multicolumn{6}{|c|}{ Well-balanced mental state ${ }^{a}$} \\
\hline not at all/little & 1 & 1 & 1 & 1 & 1 \\
\hline moderate & $0.87(0.72-1.05)$ & $1.06(0.79-1.43)$ & $1.03(0.82-1.29)$ & $1.19(1.01-1.42)$ & $0.99(0.74-1.33)$ \\
\hline much/very much & $0.92(0.78-1.08)$ & $1.09(0.84-1.41)$ & $1.15(0.95-1.40)$ & $1.14(0.98-1.32)$ & $1.07(0.83-1.38)$ \\
\hline \multicolumn{6}{|c|}{ Body mass index (BMI)a) } \\
\hline$<25$ & 1 & 1 & 1 & 1 & 1 \\
\hline $25-30$ & $1.27(1.12-1.43)$ & $0.84(0.70-1.00)$ & $0.86(0.75-0.98)$ & $0.89(0.80-0.99)$ & $0.87(0.73-1.04)$ \\
\hline$>30$ & $1.93(1.61-2.30)$ & $0.66(0.49-0.90)$ & $0.64(0.50-0.80)$ & $0.64(0.54-0.75)$ & $0.57(0.41-0.79)$ \\
\hline \multicolumn{6}{|l|}{ Health status ${ }^{b}$} \\
\hline \multicolumn{6}{|l|}{$\begin{array}{l}\text { Presence of health } \\
\text { problems/disease in } \\
\text { the past } 12 \text { months }\end{array}$} \\
\hline yes & 1 & 1 & 1 & 1 & 1 \\
\hline no & $0.75(0.66-0.85)$ & $1.26(1.02-1.56)$ & $1.38(1.17-1.62)$ & $1.22(1.08-1.38)$ & $1.48(1.19-1.84)$ \\
\hline \multicolumn{6}{|l|}{$\begin{array}{l}\text { Pain in joints } \\
\text { (last } 4 \text { weeks) }\end{array}$} \\
\hline yes & 1 & 1 & 1 & 1 & 1 \\
\hline no & $0.70(0.60-0.83)$ & $1.06(0.81-1.40)$ & $1.25(1.01-1.54)$ & $1.18(1.01-1.38)$ & $1.20(0.91-1.59)$ \\
\hline \multicolumn{6}{|l|}{ Diabetes ${ }^{c}$} \\
\hline yes & 1 & 1 & 1 & 1 & 1 \\
\hline no & $0.87(0.70-1.07)$ & $0.90(0.65-1.24)$ & $1.38(1.04-1.84)$ & $0.94(0.77-1.14)$ & $1.42(0.96-2.09)$ \\
\hline \multicolumn{6}{|l|}{ Hypertensionc) } \\
\hline yes & 1 & 1 & 1 & 1 & 1 \\
\hline no & $0.93(0.82-1.05)$ & $1.07(0.89-1.28)$ & $1.31(1.13-1.51)$ & $0.96(0.86-1.077$ & $1.23(1.02-1.48)$ \\
\hline \multicolumn{6}{|l|}{ Heart attack ${ }^{c)}$} \\
\hline yes & 1 & 1 & 1 & 1 & 1 \\
\hline no & $0.93(0.73-1.19)$ & $0.88(0.60-1.29)$ & $1.13(0.82-1.56)$ & $1.00(0.79-1.26)$ & $0.89(0.60-1.34)$ \\
\hline \multicolumn{6}{|c|}{$\begin{array}{l}\text { Health orientation a) } \\
\text { Importance of health } \\
\text { for life }\end{array}$} \\
\hline unconcerned & 1 & 1 & 1 & 1 & 1 \\
\hline health-oriented & $0.43(0.35-0.54)$ & $3.03(1.79-5.13)$ & $2.25(1.60-3.15)$ & $1.46(1.18-1.82)$ & $2.83(1.64-4.88)$ \\
\hline \multicolumn{6}{|l|}{ Mastery } \\
\hline low & 1 & 1 & 1 & 1 & 1 \\
\hline medium & $0.89(0.76-1.05)$ & $1.07(0.84-1.36)$ & $1.14(0.94-1.38)$ & $1.07(0.92-1.25)$ & $1.16(0.89-1.51)$ \\
\hline high & $0.85(0.72-1.00)$ & $0.92(0.72-1.17)$ & $1.30(1.07-1.57)$ & $1.05(0.90-1.22)$ & $1.20(0.93-1.55)$ \\
\hline \multicolumn{6}{|l|}{ Social supporta) } \\
\hline \multicolumn{6}{|l|}{ Membership in clubs } \\
\hline yes & 1 & 1 & 1 & 1 & 1 \\
\hline no & $1.85(1.64-2.07)$ & $0.68(0.58-0.80)$ & $0.48(0.42-0.55)$ & $0.93(0.84-1.02)$ & $0.51(0.43-0.60)$ \\
\hline
\end{tabular}

a) Adjusted for socio-demographic variables (age, gender, income, residential, and language region), variables on health status and BMI

b) Adjusted for socio-demographic variables (age, gender, income, residential, and language region) and variables on health status

c) Underwent medical treatment in the past 12 months

d) As defined in the text 
variables, health-orientation was strongly associated with both sports/exercise and optimal PA. Those who reported high parameter value for mastery were more likely to do vigorous sports/exercise. Belonging to any type of club was more likely to be associated with physical inactivity, and less likely with both moderate and vigorous sports/exercise and optimal PA (i. e. $\geq 210$ minutes per week).

\section{Discussion}

In 2002, the aging Swiss population consisted of approximately $\mathrm{N}=1.33$ million people aged $50-64, \mathrm{~N}=830000$ people aged $65-79$, and $\mathrm{N}=307000$ people aged $80+$, corresponding to $18.2 \%, 11.4 \%$ and $4.2 \%$ respectively of the current total population. By the year 2030, the numbers are expected to increase to approximately $\mathrm{N}=1.45$ million (50-64), 1.25 million (65-79), and $\mathrm{N}=500000$ ( $\geq 80$ ), corresponding to $19.2 \%, 16.6 \%$ and $6.7 \%$ respectively of the expected total population. This scenario implies that the $80+$ age group will be the fastest growing segment of the population, followed by the 65-79 age group.

Numerous studies have shown that PA performed throughout one's life can maintain and/or improve physical and mental health, physical functioning and quality of life, and independence (American College of Sports Medicine 1998; King et al. 1998; Spirduso \& Cronin 2001). Recently updated physical fitness recommendations for the general adult population, issued by the American College of Sports Medicine (2000), CDC (Pate et al. 1995) and the Swiss Federal Office of Sports et al. (2000), encourage people to do a certain amount of PA that will result in significant health benefits. For Switzerland and other developed countries, little is known about how physically active the older and old population is and whether the active elderly do guidelinecompliant physical activity and to what extent. Another difficulty is the fact that there is still uncertainty regarding whether or not intervention programs have led to a fundamental improvement in the physical activity behavior of aging population segments (Conn et al. 2003).

\section{Prevalence of sedentary lifestyle and of physical activity}

In developed countries, 50+ age groups were reported to be the most sedentary segment of the adult population, particularly the population aged 75+ (Breuer 2004; Martin et al. 1999; Mokdad et al. 2001; U.S. Department of Health and Human Services 1996). In the population assessed, about one-third $(32.3 \%)$ either did no sports or did insufficient habitual PA (Fig. 1). The prevalence of insufficient PA was somewhat higher than for the younger Swiss population aged 15 to $<50(26.7 \%)$. When we compared the PA be- havior of the $\geq 50$ age group to the entire Swiss population (Fig. 2) from another physical activity survey (Martin et al. 1999), we found that the older members of the population have a considerably lower prevalence of doing regular sports/ exercise but a substantially higher prevalence of doing habitual PA. However, various methodological aspects, such as the confounding effect of age in cross-sectional studies must be taken into account when judging these findings (Breuer 2003). Another important consideration is the definition of sedentary lifestyle (i. e. insufficient or no sports/PA). Since this definition may vary from survey to survey, different information will be assessed. In addition, different types of PA need to be considered. In the elderly, there are many types of PA ranging from regular sports (e. g. skiing, swimming), hiking and leisurely walking, and physical therapy to compensate for disability and physical exertion from housework and daily activities. Most surveys focus only on regular sports and endurance-based exercise. Finally, response bias may be a problem in items of recall and self-reporting. This is especially true when respondents are from older and old populations. Any of these aspects could cause bias in the findings on PA behavior as surveyed in the Swiss population aged 50 and older.

\section{Physical activity in the socio-demographic context}

Most of the relationships that we have observed between PA categories among the elderly and socio-demographic or economic variables (Tab. 1) were also reported for the entire Swiss adult population (Lamprecht \& Stamm 2000; Swiss Federal Statistical Office 2003a). The classical notion of PA behavior in the elderly is contradicted by observations that women demonstrated a higher prevalence of physical activity than men or that older age groups have a higher prevalence than younger age groups of doing habitual PA (Tab. 1 and 3). Similar findings were also reported in recent studies (Breuer 2004; King et al. 1991; Lamprecht \& Stamm 2000). A variety of popular sports/exercises designed for the older, old and oldest segments of the population and associated with new health ideals and fitness concepts, could explain the improved PA behavior among aging populations. In the context of similar findings from other studies, particularly longitudinal studies and cohort studies, our findings challenge social and health services to do some rethinking in terms of gender and age specific PA promotion as indicated in the study implications.

In light of the expected increase in the number of singleperson households among the demographically aging Swiss population (Swiss Federal Statistical Office 2003a), differing preferences for habitual PA and vigorous sports/exercise among those living in single-person households and those 
living in households with two and more persons are worthy of mention (Tab. 3). Living alone and providing for oneself requires physical exertion for daily living. The physical exertion that comes with doing housework and gardening, climbing stairs etc. with threshold energy expenditure has been proven to be health-relevant (overview see Oja 2001). Therefore, physical activity behavior in our study could have been described more realistically had this physical exertion for daily living been considered.

\section{Local environment and physical activity}

In a previous representative study, 60-74 year-old Swiss reported a preference for sports/exercise outdoors or at home rather at fitness centers (Lamprecht \& Stamm 2000). In our study, the elderly living in urban areas were less likely to lead a sedentary lifestyle and more likely to do guidelinecompliant sports/exercise, habitual PA or optimal PA of $\geq 210$ min per week than those living in rural areas (Tab. 3). This result may be less reliable since information about physically demanding work outdoors (i. e. farming and forestry) was not provided by the survey data even though such work would have had an impact on determinations of overall habitual and optimal PA. The findings may reflect the fact that infrastructures are being set up in many Swiss urban areas to encourage urban populations to walk or ride bicycles more. Greater possibilities for walking, bike rental facilities at train stations and safe bicycle paths are a few examples of this. In an Australian population segment age $60+$, access to various exercise facilities were found to be associated with PA in this sample of seniors (Booth et al. 2000). In Great Britain, the "Urbanwalks" initiative was created to enable the population to become more physically active in their everyday local environment. Such policies and programs designed to enhance the surroundings can lead to significant improvements in a community's PA behavior patterns compared to communities where such policies and programs are lacking (Brownson et al. 2001)

\section{Physical activity and health status}

In the aging segment of the Swiss population, those who reported that they were free of chronic health problems or disease and mentally well-balanced were more likely to do almost all categories of physical activity than those who suffered from health problems (Tab. 2 and 4). Although this finding was to be expected, it was surprising to find that the population segment that reported their health to be poor and/or moderate was less likely to be involved in vigorous sports/exercise, habitual PA and optimal PA than the population segment that reported themselves to be in good health, regardless of whether there were chronic health problems, pain in joints, cardiovascular risk factors or former heart attacks (Tab. 4). The apparent relevance of health perception in determining PA behavior among the older and oldest population is backed by a 55-65 year old representative population from the Swiss capital, where perceived health was strongly linked to habitual PA and self-reported fitness (Meyer et al. 2004). This finding was further backed by analyses of pooled data from 16 countries of the European Union adjusted for age, gender, education, income, and nationality, inter alia (Abu-Omar et al. 2004b).

The cross-sectional design used both in the aforementioned studies and in our study does not allow us to draw conclusions regarding causality in either direction. On one hand, health-relevant behavior (including PA) may influence an individual's perception of health (Kaplan \& Baron-Epel 2003) but an individual's perception of health may also have a positive impact on his/her health-relevant behavior (which includes sports/exercise) (Bailis et al. 2003). Additionally, the cross sectional design of our study leave open how and to which direction and extend, e. g. generation gaps natural aging might have influenced the association of health perception and health status.

Regardless of the quality of health status and health perception reported by the elderly respondents, health-orientation was a determinant for involvement in all categories of PA (Tab. 4). In this context, it is understood that older adults involved in sports/exercise programs rejected the role of being sick and that physical fitness programs can promote long-term PA sufficient to reduce disability in older adults suffering from arthritis (Minor et al. 1989). The positive association of perceived good health and health-orientation with involvement in almost every single category of PA (Tab. 4) provides an important cognitive aspect for future endeavors to get aging populations to adopt sufficient sports/ exercise habits.

\section{Implications for improving physical activity in aging populations}

This study revealed unexpected findings such as the relatively low prevalence of insufficient PA in the population of $\geq 50$ years and the relatively high prevalence of guidelinecompliant habitual PA particularly among the segment aged 65 to $80+$. However, PA is not enough according to public health recommendations. Like international physical fitness campaigns using the mass media (Bauman et al. 2000), Swiss campaigns such as "Feel Your Power" and "Getting Switzerland to move" were only partially successful: those who were already physically active became more active and those who were physically inactive failed to adopt better exercise habits (Lamprecht et al. 2004). Failing to achieve changes in 
health-relevant behavior and awareness might be one explanation (Lamprecht et al. 2004) and the different effects of various intervention approaches (information; social and behavioral; political and environmental) might be another (Dunn \& Blair 2002).

Physical activity participation in older age as a life-long process is influenced by previous life experiences. In 7098 year-old Canadians, the ability to do health-oriented sports/exercise in the latter years of their lives was significantly associated with recollections of the skills and movement capabilities gained decades earlier in childhood (Cousins 1997). These experiences match the findings on older adults for whom a positive sports/exercise experience was a greater motivating factor than the sports/exercise content itself. Having a positive experience encouraged them to stick to the fitness program or seek out other types of sports/exercise programs (Minor et al. 1989).

In our study, the relationship between PA and various socio-demographic and health-related variables suggest that a behavioral/cognitive approach should be combined with a political/environmental one for programs designed to encourage the aging Swiss population to be more physically active. Moreover, both general and individual approaches may be needed to address common health problems and physical limitations of aging populations. The clear preference that older and old Swiss have for habitual PA suggests that infrastructures for age-adequate PA should continue to be developed in both urban and rural areas. To achieve improvements in PA behavior in the Swiss population aged $50+$, involvement of multiple government agencies (not simply those dealing with health- and sports-related matters) seems indispensable. Identifying socio-demographic factors such as gender, size of household and income, and social support, inter alia, can be used to devise policies and intervention strategies to promote PA. In terms of health variables, the decision to adopt adequate PA behavior patterns seems to have less to do with whether a person actually had chronic health problems but more to do with the person's mental outlook (i. e. self-reported good health, health orientation). However, whether mental outlook drives PA or PA helps to improve mental outlook remain an open question in terms of our study results.

Future research should develop validated instruments that can be used to measure social and living environments as well as health and health orientation so that older and old populations' changing needs and unique values are addressed.

\section{Zusammenfassung}

\section{Körperliche Aktivität in der Schweizerischen Bevölkerung ab Alter 50}

Fragegestellung: Die Arbeit exploriert die Prävalenz richtlinienkonformer körperlicher Aktivität (KA) und ihre Beziehungen zu Variablen der Gesundheit und des Lebenskontextes bei Schweizer und Schweizerinnen im Alter von 50+.

Methoden: Querschnittdaten der Schweizerischen Gesundheitsbefragung 2002 wurden analysiert. Gemäss Schweizer und US-amerikanischen Richtlinien wurden vier Kategorien zur KA gebildet: moderate bzw. intensive Sportaktivität; habituelle KA; „optimale” KA. Eine fünfte Kategorie bezog sich auf sitzende Lebensweise.

Ergebnisse: Im Gesamtkollektiv von N = 8405 betrug die Prävalenz der richtlinien-konformen $\mathrm{KA}: 9,1 \%$ für moderaten Sport, 12,2\% für intensiven Sport, $45 \%$ für habituelle $K A$, $8,7 \%$ für optimale KA. Etwa $50 \%$ des Bevölkerungssegmentes

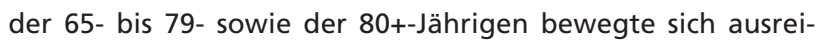

chend durch habituelle KA. Ein höherer sozioökonomischer Status stand mit moderater bzw. intensiver Sportaktivität und optimaler KA in Beziehung, ein niedrigerer Status mit habitueller KA. Leben in ländlicher und städtischer Umgebung hatte keinen differenzierenden Effekt bezüglich Prävalenz der Sportaktivität, jedoch war Leben in ländlicher Umgebung mit sitzender Lebensweise und niedriger Prävalenz habitueller bzw. optimaler KA verbunden. Unabhängig von der Existenz chronischer Gesundheitsprobleme standen die gute Gesundheit und ausgeprägte Gesundheitsorientierung mit intensiver Sportaktivität, habitueller bzw. optimaler KA in Beziehung.

Schlussfolgerung: Habituelle KA scheint das grösste Potential für die Verbesserung von gesundheitsfördernder Bewegung zu haben. Strategien zur Mobilisierung dieses Potentials liegen in den Bereichen Verhalten/Kognition sowie Politik und Umgebungsstrukturen. 
Résumé

\section{Activité physique au sein de la population suisse âgée de plus de 50 ans \\ Objectifs: Décrire l'observance des recommandations en} matière d'activité physique (AP) selon des variables se rapportant au statut socio-démographique, à l'état de santé et à l'environnement.

Méthodes: Analyse transversale basée sur les données $(\mathrm{N}=8405)$ de I'Enquête Suisse sur la Santé 2002. Quatre catégories d'AP sont utilisées: "modérée", "intensive", "habituelle" et "optimale" (cf. les directives suisses et américaines). "Mode de vie sédentaire" définit une cinquième catégorie.

Résultats: $9.1 \%$ du collectif $(\mathrm{N}=8405)$ fait partie de la catégorie "AP modérée", $12.2 \%$ de "AP intensive", $45 \%$ de "AP habituelle" et $8.7 \%$ de "AP optimale". Parmi la population âgée de 65-79 ans et de 80 ans et plus, environ $50 \%$ des personnes pratiquent une AP "habituelle". Un niveau socioéconomique élevé est associé à une d'AP "modérée", "intensive" et "optimale", tandis qu'un niveau socio-économique bas correspond à une "AP habituelle". Aucune différence entre la vie en milieu citadin ou rural n'a été mise en évidence concernant la prévalence de l'activité sportive. Par contre la vie en milieu rural est associée avec un mode de vie plus sédentaire et avec une prévalence plus basse d'AP "habituelle" et "optimale". Un bon état de santé et une orientation positive face à la santé sont en lien avec une AP "intense", "habituelle" ou "optimale", indépendamment de la présence de problèmes de santé chroniques.

Conclusions: Il faut accroître l'observance des recommandations en matière d'activité physique. Des approches comportementales, cognitives, politiques et environnementales sont suggérées pour promouvoir l'activité physique.

\section{| References}

Abu-Omar K, Rütten A, Lehtinen V (2004a). Mental health and physical activity in the European Union. Soz Praventiv Med 49: 301-9.

Abu-Omar K, Rütten A, Robine JM (2004b). Self-rated health and physical activity in the European Union. Soz Praventiv Med 49: 235-342.

American College of Sports Medicine (1998). ACSM position stand on exercise and physical activity for older adults. Med Sci Sports Exerc 30: 992-1008

American College of Sports Medicine (2000). ACSM's guidelines for exercise testing and prescription. Philadelphia.

Bailis DS, Segall A, Chipperfield JG (2003). Two views of self-rated health status. Soc Sci Med 56: 203-17.

Bauman AE, Bellew B, Owen N, Vita P (2000). Impact of an Australian mass media campaign targeting physical activity in 1998. Am J Prev Med 21: 41-7.

Booth M, Owen N, Baumann N, et al. (2000). Social-cognitive and perceived environment influences associated with physical activity in older Australians. Prev Med 31: 15-22.

Breuer C (2003). Entwicklung und Stabilität sportlicher Aktivität im Lebenslauf: zur Rolle von Alters-, Perioden- und Kohorteneffekten. Sportwissenschaft. German J Sports Sci 3: 263-79.

Breuer C (2004). Dynamics of life-span demand for sport. Sport Society 1: 50-72.
Brownson R, Baker E, Housemann R, Brennan L, Bacak $S$ (2001). Environmental and policy determinants of physical activity in the United States. Am J Public Health 91: 1995-2003.

Conn VS, Monor MA, Burks KJ, Rantz MJ, Pomeroy SH (2003). Integrative review of phyiscal activity intervention research in aging adults. J Am Geriatr Soc 51: 1159-68.

Cousins SO (1997). Elderly tomboys? Sources of self-efficacy for physical activity in later life. $\mathrm{J}$ Aging Phys Activity 5: 229-43.

Crimmins E, Hayward M, Saito Y (1994). Changing mortality and morbidity rates and the health status and life expectancy of the older population. Demography 31: 159-75.

Cunningham GO, Michael YL (2004). Concepts guiding the study of the impact of the built environment on physical activity for older adults: a review of the literature. Am J Health Promot 18: 435-43.

Dunn AL, Blair SN (2002). Translating evidenced-based physical activity interventions into practice: the 2010 Challenge. Am J Prev Med 22: $221-5$.

Kaplan G, Baron-Epel O (2003). What lies behind the subjecitve evaluation of health status? Soc Sci Med 56: 1669-76.

King AC, Haskell WL, Taylor CB, Kraemer HC, DeBusk RF (1991). Group-versus home-based exercise training in healthy older men and women: a community-based clinical trial. J Am Med Assoc 266: 1535-42.

King AC, Rejeski WJ, Buchner DM (1998). Physical activity interventions targeting older adults: a critical review and recommendations. Am J Prev Med 15: 316-33.
Lamprecht M, Fischer A, Ruckstuhl B, Stamm $H P$ (2004). "Feel Your Power": potentials and limits of a health promotion campaign. Schweiz Z Sportmed Sporttraumatol 52: 71-7.

Lamprecht M, Stamm HP (2000). Sports Switzerland 2000: sport activity and Sport consumption in the Swiss population. Basel: Swiss Olympic Association.

Martin BW, Mäder U, Calmonte R (1999). Physical activity related attitudes, knowledge and behaviour in the Swiss Population: results of the HEPA survey 1999. Schweiz Z Sportmed Sporttraumatol 47: 165-9.

Meyer K, Niemann S, Abel T (2004). Gender differences in physical activity and fitness: association with self-reported health and healthrelevant attitudes in a middle-aged Swiss urban population. J Public Health 12: 283-90.

Minor M, Hewett J, Webel R, Anderson S, Kay D (1989). Efficacy of physical conditioning exercise in patients with rheumatoid arthritis and osteoarthritis. Arth Rheumat 32: 1396-405.

Mokdad AH, Bowman BA, Ford ES, Vinicor F, Marks JS, Koplan JP (2001). The continuing epidemics of obesity and diabetes in the United States. JAMA 286: 1195-201.

Oja P (2001). Dose response between total volume of physical activity and health and fitness. Med Sci Sports Exerc 33: 428-37.

Pate RR, Pratt M, Blair SN, et al. (1995). Physical activity and public health: a recommendation from the Centers for Disease Control and Prevention and the American College of Sports Medicine. JAMA 273: 402-7. 
Spirduso WW, Cronin DL (2001). Exercise doseresponse effects on quality of life and independent living in older adults. Med Sci Sports Exerc 33: 598-608

Stewart A, Ware J, Brook R (1977). The meaning of health: understanding functional limitations. Medical Care 15: 939-52.

Swiss Federal Office of Sports, Swiss Federal Office of Health, Swiss Federal Statistical Office, Network Health and Exercise Switzerland (2000). Physical activity in the Swiss population: level and associations with health. Schweiz Z Sportmed Sporttraumatol 48: 87-8.
Swiss Federal Statistical Office (2003a). Census 1970-2000: press communication. Neuchâtel: SFSO.

Swiss Federal Statistical Office (2003b). Swiss Health Survey 2002. Neuchâtel: SFSO.

U.S. Department of Health and Human Services (1996). Physical activity and health: a report of the Surgeon General. Atlanta, GA: U.S. Department of Health and Human Services, Centers for Disease Control and Prevention, National Center for Chronic Disease Prevention and Health Promotion.

Vita A, Terry R, Hubert H, Fries J (1998). Aging, health risks, and cumulative disability. N Engl J Med 338: 1035-41.
Address for correspondence

PD Dr. Katharina Meyer, PhD, MPH Swiss Health Observatory \& University of Bern

Espace de l'Europe

CH-2010 Neuchâtel

Tel.: +4132 7136536

e-mail: meyer.katharina@bluewin.ch 